\title{
EVALUATION OF APÉRY-LIKE SERIES THROUGH MULTISECTION METHOD
}

\author{
Wenchang Chu AND Flavia LuCia Esposito
}

\begin{abstract}
By combining the multisection series method with the power series expansion of arcsin-function, we investigate Apéry-like infinite series involving the central binomial coefficients in denominators. By constructing and resolving systems of linear equations, numerous remarkable infinite series formulae (generated by using an appropriate computer algebra system) for $\pi$ and special values of the logarithm function are established, including some recent results due to Almkvist et al. (2003) and Zheng (2008).
\end{abstract}

Mathematics subject classification (2010): Primary 11Y60, Secondary 14G10.

Keywords and phrases: Multisection series, Apéry-like series, central binomial coefficient.

\section{REFERENCES}

[1] G. Almkvist et al., Some new formulas for $\pi$, Experimental Mathematics 12 (2003), 441-456.

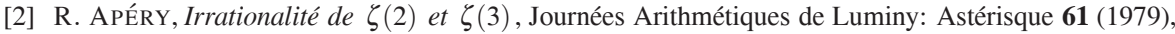
$11-13$.

[3] D. H. BAILEY et al., The quest for $\pi$, Mathematical Intelligencer 19:1 (1997), 50-57; MR1439159 (98b:01045).

[4] N. D. BARUAH et al., Ramanujan's series for $1 / \pi$ : a survey, American Mathematical Monthly 109:7 (2009), 567-587.

[5] J. M. Borwein et al., Central binomial sums, multiple Clausen values and zeta values, Experimental Mathematics 10 (2001), 25-34.

[6] J. M. Borwein AND R. Girgensohn, Evaluations of binomial series, Aequationes Mathematicae 70 (2005), 25-36.

[7] D. BRADley, More Apéry-like formulae: On representing values of the Riemann zeta function by infinite series damped by central binomial coefficients, preprint, 2002; http://www. math. umaine.edu/ bradley/papers/bivar5.pdf.

[8] W. CHu, Hypergeometric series and the Riemann zeta function, Acta Arithmetica 82 (1997), 103-118.

[9] W. CHU, Symmetric functions and the Riemann Zeta series, Indian Journal of Pure and Applied Mathematics 31:12 (2000), 1677-1689; MR2002e:11112.

[10] W. CHU, Dougall's bilateral ${ }_{2} \mathrm{H}_{2}$-series and Ramanujan-like $\pi$-formulae, Mathematics of Computation 80:276 (2011), 2223-2251.

[11] W. CHU AND D. Y. ZHENG, Infinite series with harmonic numbers and central binomial coefficients, International Journal of Number Theory 5:3 (2009), 429-448.

[12] L. Comtet, Advanced Combinatorics, Reidel, Boston, Massachusetts, 1974.

[13] C. ElSnER, On sums with binomial coefficient, Fibonacci Quarterly 43:1 (2005), 31-45.

[14] M. GENČEv, Binomial sums involving harmonic numbers, Mathematica Slovaca 61:2 (2011), 215 226.

[15] M. L. Glasser, A generalized Apéry series, Journal of Integer Sequences 15 (2012), \#Article 12.4.3.

[16] J. Guillera, Some binomial series obtained by the WZ-method, Advances in Applied Mathematics 29:4 (2002), 599-603.

[17] J. GUILlERA, Hypergeometric identities for 10 extended Ramanujan-type series, Ramanujan Journal 15:2 (2008), 219-234. 
[18] D. H. LEHMER, Interesting series involving the central binomial coefficient, American Mathematical Monthly 92 (1985), 449-457.

[19] H. MuZAFFAR, Some interesting seires arising from the power series expansion of $\left(\sin ^{-1} x\right)^{q}$, International Journal of Mathematics and Mathematical Sciences 14 (2005), 2329-2336.

[20] A. J. VAN DER POORTEN, A proof that Euler missed ..., Mathematical Intelligencer 1 (1979), 195203.

[21] T. SHERMAN, Summations of Glaisher and Apéry-like numbers, available at http://math.arizona.edu/ ura/001/sherman.travis/series.pdf.

[22] L. ZHANG AND W. JI, The series of reciprocals of non-central binomial coefficients, American Journal of Computational Mathematics 3 (2013), 31-37.

[23] B. SURY et al., Identities involving reciprocals of binomial coefficients, Journal of Integer Sequences 7 (2004), \#Article 4.2.8.

[24] D. Y. ZHENG, Multisection method and further formulae for $\pi$, Indian Journal of Pure and Applied Mathematics 39:2 (2008), 137-155.

[25] I. J. ZUCKER, On the series $\sum_{k=1}^{\infty}\left(\begin{array}{c}2 k \\ k\end{array}\right)^{-1} k^{-n}$, Journal of Number Theory 20:1 (1985), 92-102. 